\title{
The Critical Role of Tissue Angiotensin-converting Enzyme as Revealed by Gene Targeting in Mice
}

\author{
Charles R. Esther, Jr., ${ }^{\star}$ Elaine M. Marino,, Tom E. Howard, Annie Machaud, ${ }^{\ddagger}$ Pierre Corvol,‡ Mario R. Capecchi, ${ }^{\S}$ \\ and Kenneth E. Bernstein* \\ *Department of Pathology, Emory University, Atlanta, Georgia 30322; $\doteqdot$ Institute National de la Santé et de la Recherche Medicale Unit 36, \\ College de France, Paris, France 75005; and ${ }^{\S}$ Howard Hughes Medical Institute, Eccles Institute of Human Genetics, University of Utah, \\ Salt Lake City, Utah 84112
}

\begin{abstract}
Angiotensin-converting enzyme (ACE) generates the vasoconstrictor angiotensin II, which plays a critical role in maintenance of blood pressure in mammals. Although significant ACE activity is found in plasma, the majority of the enzyme is bound to tissues such as the vascular endothelium. We used targeted homologous recombination to create mice expressing a form of ACE that lacks the $\mathrm{COOH}$-terminal half of the molecule. This modified ACE protein is catalytically active but entirely secreted from cells. Mice that express only this modified ACE have significant plasma ACE activity but no tissue-bound enzyme. These animals have low blood pressure, renal vascular thickening, and a urine concentrating defect. The phenotype is very similar to that of completely ACE-deficient mice previously reported, except that the renal pathology is less severe. These studies strongly support the concept that the tissue-bound ACE is essential to the control of blood pressure and the structure and function of the kidney. (J. Clin. Invest. 1997. 99:2375-2385.) Key words: peptidyl-dipeptidase A • blood pressure - kidney • urine concentration $\bullet$ male fertility
\end{abstract}

\section{Introduction}

Angiotensin-converting enzyme $(\mathrm{ACE})^{1}$ plays a central role in the renin-angiotensin system through generation of the peptide angiotensin II from its inactive precursor angiotensin I (1). Angiotensin II induces vasoconstriction, aldosterone release, and other physiologic actions that act to raise blood pressure (2). ACE inhibitors block the formation of angiotensin II and have been used to treat hypertension and heart failure.

Cloning of ACE showed that this molecule is a single polypeptide chain that contains two homologous domains,

C.R. Esther, Jr. and E.M. Marino contributed equally to this work.

Address correspondence to Kenneth E. Bernstein, M.D., Department of Pathology, Room 7107A WMB, Emory University, Atlanta, GA 30322. Phone: 404-727-3134; FAX: 404-727-8540; E-mail: kbernst @emory.edu

Received for publication 24 January 1997 and accepted in revised form 6 March 1997.

1. Abbreviations used in this paper: ACE, angiotensin-converting enzyme; AcSDKP, $N$-acetyl-seryl-aspartyl-lysyl-proline; ES cells, embryonic stem cells; HGG, hippuryl-glycyl-glycine; HHL, hippuryl-histidyl-leucine; neo ${ }^{\mathrm{r}}$, neomycin resistance.

J. Clin. Invest.

(C) The American Society for Clinical Investigation, Inc.

0021-9738/97/05/2375/11 \$2.00

Volume 99, Number 10, May 1997, 2375-2385 each with an independent catalytic site (3-5). Each catalytic site is active against angiotensin I and other peptide substrates. While the two catalytic sites generate angiotensin II with equal efficiency, they vary in a number of biochemical parameters including the ability to hydrolyze peptide substrates other than angiotensin I (6). For example, the carboxy-terminal domain requires a higher chloride concentration for efficient hydrolysis of some substrates such as hippuryl-histidyl-leucine (HHL) and hippuryl-glycyl-glycine (HGG). Furthermore, leutinizing hormone-releasing hormone and the hematopoetic stem cell inhibiting peptide $N$-acetyl-seryl-aspartyl-lysyl-proline (AcSDKP) are cleaved more efficiently by the amino terminal catalytic site than by the carboxyl-terminal active site $(7,8)$. Although the amino-terminal domain does cleave AcSDKP in vivo (9), the physiological significance of the two catalytic sites is not at present known.

The vast majority of ACE enzymatic activity is associated with tissues such as the vascular endothelium and the proximal tubular epithelium of the kidney. ACE is an ectoenzyme; both catalytic domains are found outside of cells, while the protein is bound to cell membranes by a hydrophobic anchor sequence found at the carboxyl terminus (1). ACE activity is also found in a soluble form within plasma resulting from the enzymatic cleavage of tissue-bound ACE (10). The functional significance of circulating ACE versus tissue-bound ACE has been of great interest since 1967 when $\mathrm{Ng}$ and Vane first emphasized the physiologic role of lung ACE activity in the conversion of angiotensin I to angiotensin II (11). Others have failed to correlate the antihypertensive effects of ACE inhibitors with the degree of plasma ACE inhibition (12). These data support the hypothesis that tissue-bound ACE activity plays a major role in the production of angiotensin II. Furthermore, such studies suggest that tissue-bound ACE may participate in local renin-angiotensin systems that function independently from the circulating renin-angiotensin system (13).

A unique form of ACE is found in the testis. This isozyme is called testis ACE, and it is transcribed in postmeiotic male germ cells using a unique testis-specific promoter located within the 12th intron of the somatic $A C E$ gene $(14,15)$. Testis ACE is about half the size of somatic ACE and contains only the carboxyl-terminal catalytic domain. Recent studies of ACE-deficient mice have suggested that testis ACE plays an important role in male fertility $(16,17)$.

Previous studies of ACE deficient mice by Krege et al. (16) and our lab (17) were unable to distinguish between the effects of somatic ACE and testis ACE since the animals lacked both isozymes. To address this issue, we attempted to create a mouse strain expressing only the somatic form of ACE. We succeeded in generating mice that lack testis $\mathrm{ACE}$ and have a partial restoration of plasma somatic ACE. However, the somatic ACE in these animals is truncated and contains only the amino-terminal active site. More importantly, this form of 
ACE lacks the membrane-spanning anchor region and is therefore found only as a soluble enzyme. Although this soluble enzyme can generate angiotensin II efficiently, these animals (now termed ACE.2) have very low blood pressure, renal vascular thickening, an inability to concentrate urine, and reduced male fertility. This phenotype is very similar to that of our ACE.1 mice, which completely lack all ACE (17). This similarity between ACE.1 and ACE. 2 mice supports the hypothesis that tissue-bound ACE is critically important in the physiologic generation of angiotensin II.

\section{Methods}

\section{Creation of mice with a mutant ACE allele}

The DNA construct used for targeted homologous recombination was a modification of a previously described targeting construct (17). The previous construct consisted of two arms of $A C E$ genomic DNA flanking a $3.1-\mathrm{kb}$ neomycin cassette. One arm contained $6.9 \mathrm{~kb}$ of $A C E$ genomic sequence extending from the middle of somatic $A C E$ exon 1 to $91 \mathrm{bp} 5^{\prime}$ of the testis $A C E$ transcription start site. The second arm was $1.8 \mathrm{~kb}$ of genomic $A C E$ sequence extending from $17 \mathrm{bp}$ downstream of the testis $A C E$ transcription start site to the middle of somatic $A C E$ exon 15. In this construct, the testis $A C E$ translation start site was changed from ATG to TTG. The resulting construct is missing the DNA sequence between $-91 \mathrm{bp}$ and +17 bp relative to the testis $A C E$ transcription start site. A 1.8-kb thymidine kinase cassette was inserted $3^{\prime}$ to the other fragments.

The targeting construct used for the ACE.2 mice differs from the previous construct in having a $2.2-\mathrm{kb}$ fragment of $A C E$ cDNA inserted into MluI and EcoRV sites immediately $5^{\prime}$ to the neomycin cassette. This cDNA fragment was generated by PCR from cloned $A C E$ cDNA with the primers 5' - CAGAACGCGTGCAGACCTAGAGACTGATGAAGC-3' and 5'-CAGACGATATCGACCTTTAATTGTATTCACAG-3'. The fragment incorporates $2.2 \mathrm{~kb}$ of $A C E$ cDNA sequence including all of the somatic $A C E$ coding exons (13-25) downstream of the neomycin cassette in the previous construct and 216 bp of $3^{\prime}$ untranslated region including a poly-adenylation site. The cDNA fragment contains a consensus $3^{\prime}$ splice acceptor sequence (GCAG) immediately $5^{\prime}$ of the coding region.

The DNA construct was linearized with NotI and electroporated into R1 ES cells. The isolation of targeted homologous recombinants and the generation of chimeric heterozygotes and homozygous mice was performed as previously described (18). Genotyping was performed by Southern blot and PCR analyses (17).

\section{ACE assays}

Plasma was collected either by retroorbital bleed into heparinized capillary tubes or via heparinzed syringe from the inferior vena cava of animals anesthetized with metofane. Tissues were taken from anesthetized animals, frozen in liquid nitrogen, and stored at $-80^{\circ} \mathrm{C}$ until needed. The tissues were then homogenized in ACE homogenization buffer ( $50 \mathrm{mM}$ Hepes, $\mathrm{pH} 7.4,150 \mathrm{mM} \mathrm{NaCl}, 0.5 \%$ Triton X-100, $25 \mu \mathrm{M} \mathrm{ZnCl}_{2}, 1 \mathrm{mM}$ PMSF) and clarified by centrifugation for $15 \mathrm{~min}$ at $10,000 \mathrm{~g}$. ACE activity against the artificial substrate hippuryl-glycyl-glycine was determined using the ACE-DIRECT REA (ALPCO, Windham, NH). For this assay, plasma was diluted 1:4 and tissue diluted to $30 \mu \mathrm{g}$ protein per assay in reaction buffer. All samples were measured in duplicate.

Initial velocities of wild-type ACE $(+/+)$ and homozygous mutant ACE $(-/-)$ were determined using HHL, AcSDKP and angiotensin I as substrate over a concentration range of 0.02 to $1.25 \mathrm{mM}$. Assays were performed in a pool of plasma of $+/+$ and $-/-$ mice, diluted $1 / 50$ and $1 / 25$, respectively. In addition, angiotensin I to angiotensin II conversion was measured in individual plasma samples. The rate of hydrolysis of all substrates used was quantified by HPLC on a Waters apparatus consisting of 600 controllers (Waters Co., Milford,
MA), absorbance detectors 486 and 717 autosampler or 712 WISP directed by a Millenium chromatography manager.

$H H L$. Assay was performed in $300 \mathrm{mM}$ chloride as described by Cushman and Cheung (19) and the released Hip was quantified by HPLC as previously described (5). The optimal chloride concentration for substrate hydrolysis was determined as previously described (5).

AcSDKP. Reactions were performed in $100 \mathrm{mM}$ Hepes, $\mathrm{pH} 7.0$, $50 \mathrm{mM} \mathrm{NaCl}, 10 \mu \mathrm{M} \mathrm{ZnSO}_{4}$, and terminated by the addition of $0.1 \%$ trifluoracetic acid (final concentration). The rate of hydrolysis of AcSDKP was determined by measuring the production the terminal Lys-Pro, which was resolved and quantified under isocratic conditions (water: acetonitrile [98:2], 0.1\% TFA). Unhydrolyzed AcSDKP was eluted and quantified by increasing the concentration of acetonitrile to $10 \%$. The products were resolved by reverse-phase HPLC, on a $5-\mu \mathrm{m}$ Puresil C18 column (Waters Co.) at a flow rate of $1 \mathrm{ml} / \mathrm{min}$. Retention times of KP and AcSDKP were 4.7 and $13.2 \mathrm{~min}$, respectively, at a detection limit of $0.2 \mathrm{nmol}$ at $200 \mathrm{~nm}$.

Angiotensin I. Reactions were performed in $50 \mathrm{mM}$ Hepes $\mathrm{pH}$ $7.5,50 \mathrm{mM} \mathrm{NaCl}, 10 \mu \mathrm{M} \mathrm{ZnSO}_{4}$, and stopped by the addition of $2 \%$ $\mathrm{H}_{3} \mathrm{PO}_{4}$ (final concentration). Angiotensin II and angiotensin I were resolved and quantified by isocratic reverse-phase HPLC in $18 \%$ (vol/vol) acetonitrile, $86 \mathrm{mM}$ triethylammonium phosphate, $\mathrm{pH} 3.0$, at a flow rate of $1 \mathrm{ml} / \mathrm{min}$ on a $3-\mu \mathrm{m}$ Hypersil C18 column (Merck EM Science, Gibbstown, NJ) at $45^{\circ} \mathrm{C}$. Retention times of angiotensin II and angiotensin I were 3.4 and $7.0 \mathrm{~min}$, respectively, at a detection limit of $0.5 \mathrm{nmol}$ at $200 \mathrm{~nm}$.

\section{Northern blot}

Poly A+ RNA was isolated from the lungs and kidneys of one wild type, one heterozygous, and one homozygous mutant mouse using guanidine isothiocyanate (20) and oligo dT cellulose from the mRNA purification kit by Pharmacia (Uppsala, Sweden). Aliquots of mRNA were separated on a formaldehyde agarose gel, transferred to nylon and $A C E$ mRNA detected with ${ }^{32} \mathrm{P}$-labeled probe made from full length $A C E$ cDNA (4).

cDNA was prepared using poly A+ RNA and the Timesaver, cDNA Synthesis kit from Pharmacia. A portion of $A C E$ cDNA between positions 1758 and 2531 was amplified from the total cDNA by PCR using primers ACE.36 (5'-AGGTGTTGCAGGCTGGCTG-3') and ACE.85 (5'-AGGTTGTCAGACTCGTA-3'). The resulting product was cloned into the TA cloning vector from Invitrogen (San Diego, CA) and sequenced with the Sequenase 2.0 kit (Amersham Corp., Arlington Heights, IL).

\section{Western blot}

Tissue samples for Western blot were homogenized in RIPA buffer (20 mM Tris pH 7.4, 2.5 mM EDTA, 1\% Triton X-100, 10\% glycerol, $1 \%$ deoxycholic acid, $0.1 \%$ SDS, $1 \mathrm{mM}$ PMSF, $10 \mathrm{ng} / \mathrm{ml}$ Aprotinin). $20 \mathrm{mg}$ of protein was run by SDS-PAGE on a $7.5 \%$ gel, and the separated proteins transferred to PVDF or nitrocellulose membranes. The membranes were probed with a rabbit polyclonal anti-mouse ACE antibody, and developed with an enhanced chemiluminescence kit from Amersham Corp. (21).

\section{Histology}

All samples for histological analysis were preserved in Bouin's fixative or $10 \%$ formalin for at least $3 \mathrm{~d}$. They were then embedded in paraffin and prepared using standard methods.

\section{Blood pressure}

Systolic blood pressures of the mice were determined using an automated tail cuff system as described by Krege et al. (22). Training measurements were taken for $7 \mathrm{~d}$ to acclimatize the animals to the machine, followed by $4 \mathrm{~d}$ of recorded measurements. On each day, three sets of 10 measurements were taken for each mouse and the first set discarded. To eliminate bias caused by struggling animals or other difficulties, each set of measurements was accepted only if the standard deviation of the set was less than or equal to $9 \mathrm{mmHg}$. 


\section{Urine collection}

For metabolic studies, all animals were pair-fed for $3 \mathrm{~d}$. Urine was collected from animals of the same age and gender for $24 \mathrm{~h}$ in a metabolic cage without food or water. Mineral oil was placed in the urine collection chamber to minimize evaporation. Water was removed for a total of $30 \mathrm{~h}$ including $6 \mathrm{~h}$ before the animals were placed in the metabolic cage. After $24 \mathrm{~h}$, urine was collected, spun for $5 \mathrm{~min}$ at $5,000 \mathrm{rpm}$ to remove particulate matter and frozen at $-20^{\circ}$ until analysis.

Urine osmolality and electrolytes were determined by standard methods using the Wescat 5500 Vapor Pressure Osmometer (Wescor, Inc., Logan, UT).

\section{Superovulation}

Wild-type, female C57BL/6J mice were purchased from Harlan (Indianapolis, IN) and superovulated when 5-6 wk old. Superovulation was induced by intraperitoneal injection of $5 \mathrm{U}$ of pregnant mares serum (gonadotrophin) between 1 and 3 p.m. followed by injection of $5 \mathrm{U}$ of human chorionic gonadotrophin $46 \mathrm{~h}$ later. Both hormones were purchased from Sigma Chemical Co. (St. Louis, MO). Superovulated females were housed individually with single males beginning the evening after the second injection. The following morning the males were removed, and the females checked for copulatory plugs. Females were killed during the last week of gestation, and the number of pups present in the uterus was counted.

\section{Results}

Creation of homozygous mutant mice. The genetic modification of the $A C E$ locus was achieved using targeted homologous recombination in embryonic stem (ES) cells with the replacement construct shown in Fig. $1 A$. This construct was designed to replace a region extending from $91 \mathrm{bp}$ upstream of the testis $A C E$ transcription start site through the first $17 \mathrm{bp}$ of the testis $A C E$ first exon with a neomycin cassette. Also, the testis $A C E$ transcription start site was changed from ATG to TTG. In a previously published study, we showed that these modifications eliminated testis ACE production in homozygous mutant mice (17). We also found that these modifications of the testis $A C E$ promoter region eliminated production of somatic ACE. To restore production of somatic ACE, a cDNA element containing all of the somatic $A C E$ coding regions downstream of the neomycin cassette (exons 13 through

A

Wild-Type Allele

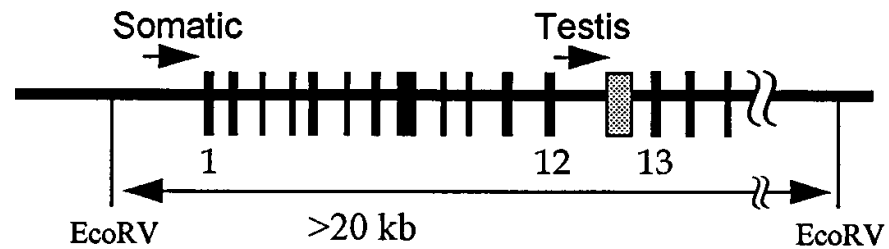

Construct

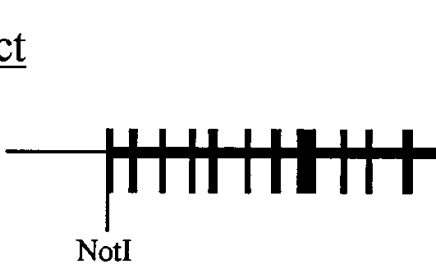

Modified Allele
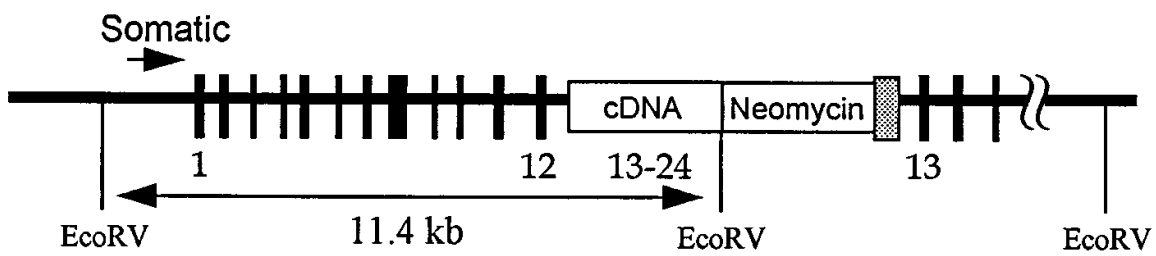

No Testis

Promoter
ATG Altered

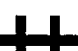
TK EcoRV EcoRV
B

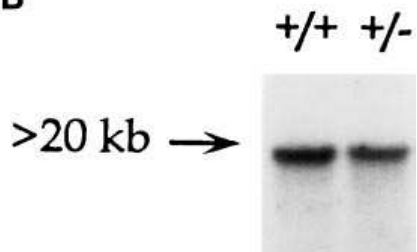

$11.4 \mathrm{~kb}$

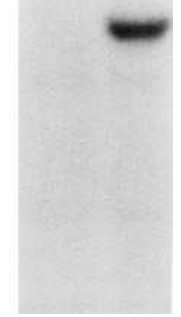

Figure 1. Targeted disruption of the ACE allele. $(A)$ The top shows the wild-type ACE allele, with exons as filled boxes and the testis specific exon in gray. The somatic and testis ACE promoters are represented by arrows, and restriction sites are labeled below. The middle shows the targeting construct. This is composed of ACE genomic sequence from the middle of somatic ACE exon 1 to the middle of somatic ACE exon 15 in which the testis ACE promoter has been deleted and replaced with a neomycin resistance cassette. A cDNA element encoding exons 13-25 of somatic ACE is inserted 5' to the neomycin resistance cassette. The construct was designed to eliminate testis ACE but allow for the production of somatic ACE despite the presence of the neomycin resistance cassette in the middle of the ACE gene. A thymidine kinase (TK) cassette at the $3^{\prime}$ end of the construct allowed a positive/negative selection for homologous recombinants. The bottom shows the modified ACE allele after targeted homologous recombination. An additional EcoRV restriction site is present in the modified allele. $(B)$ Southern blot analysis of ES cells. Genomic DNA was isolated from normal $(+/+)$ and successfully targeted $(+/-)$ stem cells, digested with EcoRV, separated by agarose electrophoresis and blotted. The blot was probed with a 1-kb portion of cloned genomic DNA immediately 5' of somatic ACE exon 1 (17). The probe hybridizes to $\mathrm{a}>20-\mathrm{kb}$ fragment from the wild type allele and an 11.4-kb fragment from the modified allele. 
25) was inserted into intron 12 upstream of all other modifications. It was hoped that splicing of this cDNA into the somatic $A C E$ transcript would provide all of the genetic information needed to produce full length somatic ACE.

Construct DNA was electroporated into R1 ES cells, and putative homologous recombinants were isolated by positive and negative selection. Proper homologous recombination was verified by Southern blot (Fig. $1 \mathrm{~B}$ ). ES cells containing the modified $A C E$ locus were injected into blastocysts of C57BL/6 mice. Chimeric offspring were screened for incorporation of the modified allele by PCR and mice bearing the modified allele were bred to C57BL/6 females to produce the F1 generation. F1 animals were interbred to produce $\mathrm{F} 2$ generation mice that were either wild-type $(+/+)$, heterozygous $(+/-)$, or homozygous mutant ( $-/-$ ) for the $A C E$ locus. This strain of mice was designated ACE. 2 to distinguish it from the ACE-defi- cient ACE.1 strain animals previously characterized (17). Of the 202 F2 mice genotyped, 44 were wild-type, 121 were heterozygous, and 32 were homozygous mutant.

Genetic and biochemical analysis. Production of normal somatic ACE from the modified allele could occur only if the cDNA element is spliced correctly into the somatic $A C E$ transcript. To investigate splicing from the modified allele, mRNA was isolated from the lung and kidneys of wild-type, heterozygous, and homozygous mutant mice. Northern blot analysis for $A C E$ mRNA demonstrated that the homozygous mutant mice produced small amounts of a transcript that appeared similar in size to the smaller of the two naturally occurring somatic $A C E$ transcripts (Fig. $2 A$ ). The presence of a single transcript from the modified allele is expected since the construct contains only one of the two polyadenylation sites that generate two transcripts in normal animals (4). To further examine
A

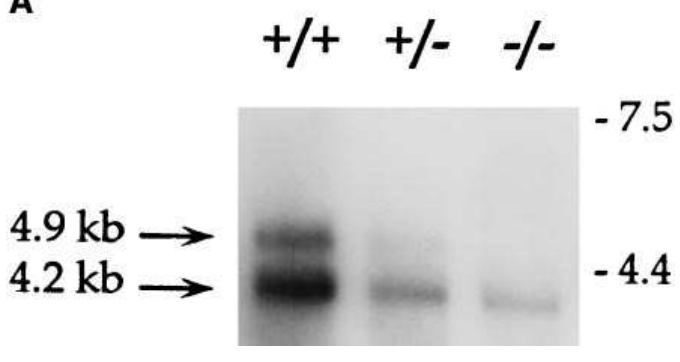

B

\section{Splicing}

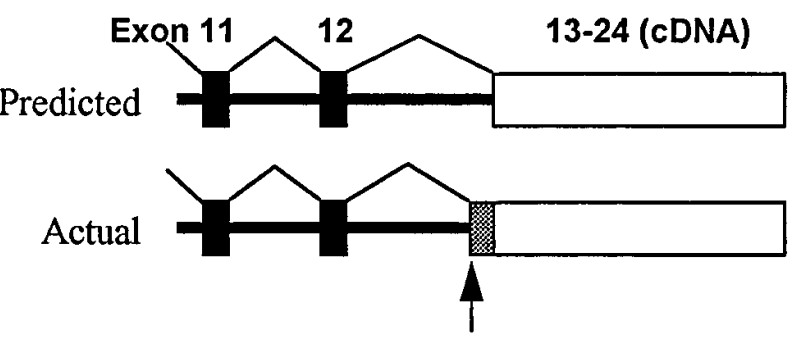

Cryptic Splice Site

\section{Spliced mRNA}

Predicted
Exons 1-12 13-24 (cDNA)

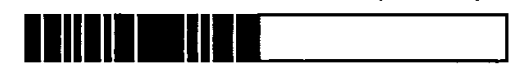

Actual

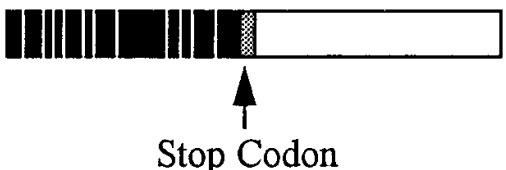

\section{Protein Structure}

Predicted

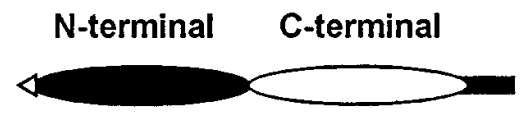

\section{Actual}

Figure 2. Northern blot and sequencing of modified ACE transcript. $(A)$ Poly A+ RNA was isolated from the combined lung and kidneys of a wild-type $(+/+)$, heterozygous $(+/-)$, and homozygous mutant $(-/-)$ mouse. After electrophoresis, the poly A+ RNA was transferred to a nylon membrane, and hybridized to a probe made from full-length ACE cDNA (4). More poly A+ RNA from the $-1-$ mouse was loaded compared to the other genotypes ( $4 \mathrm{vs} .2 \mu \mathrm{g}$ ) to compensate for reduced signal. The wild type mouse contained the expected two ACE bands of 4.2 and $4.9 \mathrm{~kb}$. The heterozygous animal had reduced levels of the two bands, and the homozygous mutant had a low level of only the smaller of the two bands. $(B)$ The top shows the predicted and actual splicing of the modified ACE gene between the naturally occurring exons (black boxes) and the cDNA element (white box). The resulting mRNA contains an extra 40 nt of sequence (gray box) between exons 1-12 and exons 13-24 from the cDNA (middle). This encodes an in-frame stop codon just three amino acids downstream of exon 12 . The normal protein structure of somatic ACE (bottom) consists of two homologous domains (ovals) with an $\mathrm{NH}_{2}$-terminal signal sequence (triangle) and a COOH-terminal hydrophobic membrane anchor (black bar). Because of the stop codon, the actual protein structure from the modified allele transcript consists of only the signal sequence and $\mathrm{NH}_{2}$-terminal domain. 
splicing, the region spanning the potential splice junction between exon 12 and the cDNA element was cloned by RT-PCR from homozygous mutant mice. DNA sequencing of the cloned fragment showed that the somatic transcript from the modified allele contained a 40 nucleotide insertion between exon 12 and the cDNA element (Fig. 2 B). This insertion resulted from splicing to a cryptic splice acceptor site (tctctcag $^{\mathbf{7} G G T C C T A G)}$ located 40 bp upstream of the cDNA element within the twelfth intron. The insertion introduced an in-frame stop codon (TAG) just two amino acids after the last codon of exon 12. Therefore, although transcription from the modified allele produced a full-length mRNA, translation was predicted to terminate halfway through the transcript. To test this, blood from wild-type, heterozygous, and homozygous mutant animals was collected, and plasma was analyzed by Western blot with a polyclonal anti-ACE antibody (Fig. $3 A$ ). A single somatic ACE band was present in homozygous mutant animals at a molecular mass of $85 \mathrm{kD}$. This size is roughly half that of normal somatic ACE from wild-type mice. Heterozygous mice contained both normal and modified forms of somatic ACE. These data are consistent with the predictions of the RNA sequence analysis.

The truncation of ACE has two important consequences. First, the modified protein contains only the amino terminal domain of somatic ACE. Second, the modified protein lacks the carboxyl terminal hydrophobic tail that anchors somatic ACE to the plasma membrane of cells. Thus, the modified protein should be entirely secreted into plasma, resulting in little or no tissue-bound ACE activity. Examination of lung, kidney, and testis by Western blot confirmed that no ACE protein was present in homozygous mutant animals (Fig. $3 \mathrm{~B}$ ). The Western blot also showed that the testis specific isoform of ACE is also absent in these mice.

To further examine the characteristics of the modified ACE protein, ACE enzyme activity was measured in plasma from the three genotypes using the commercially available artificial substrate hippuryl-glycine-glycine (HGG). With this substrate, the homozygous mutant mice have about $15 \%$ the activity of wild-type animals (Fig. $4 A$ ). Lung, kidney, and testis from these animals were also analyzed for ACE activity. Consistent with Western blot analysis, no ACE activity was detected in any tissue from homozygous mutant mice (Fig. $4 B$ ).

Interpretation of these results is complicated by the modified somatic ACE protein found in the homozygous mutant animals. The amino terminal domain of ACE differs from the carboxyl terminal domain in its affinity and catalytic activity towards various substrates. This is particularly true for the small substrates, as shown in Fig. 4 C. Here, pooled plasma from each of the two genotypes was tested against substrates for which the individual catalytic activity of the two ACE domains is known. The initial catalytic velocities were determined in zero order (substrate not limiting) conditions. AcSDKP is a naturally occurring amino-terminal specific substrate (8). This peptide was cleaved by plasma from homozygous mutant mice at rate $89 \%$ of that of wild-type plasma. In contrast, the artificial substrate, HHL is almost exclusively an ACE carboxy-terminal substrate in presence of high chloride concentration (300 mM) (5). Under these conditions, ACE activity in homozygous mutant plasma towards this substrate was only $6 \%$ of wild type, consistent with the results with HGG. The chloride sensitivity of ACE activity in plasma was also tested. The carboxy-terminal domain exhibits a large activation by
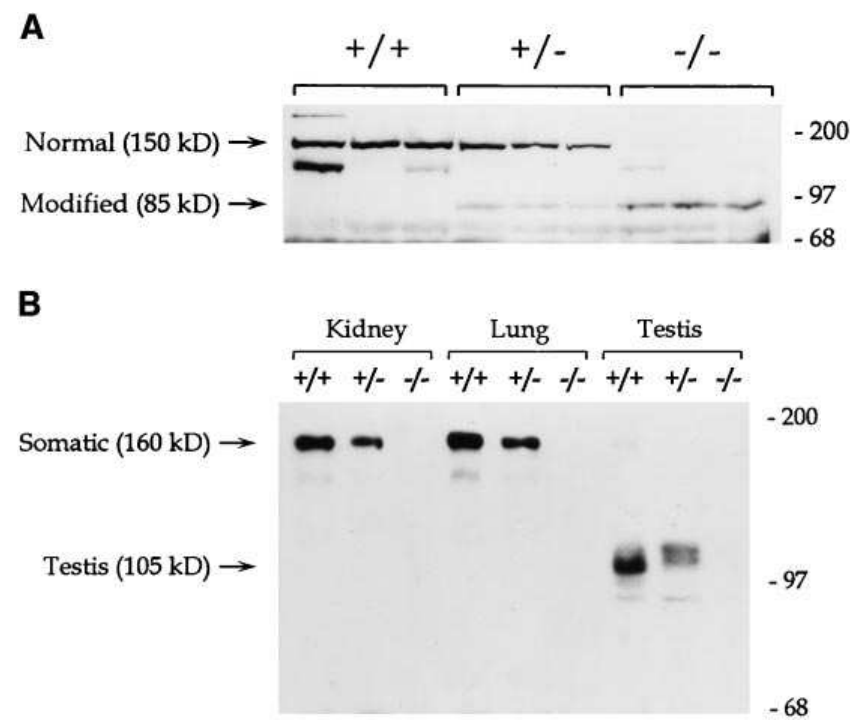

Figure 3. Western blot. (A) $20 \mu \mathrm{g}$ of protein from plasma of wildtype $(+/+)$, heterozygous $(+/-)$, and homozygous mutant $(-/-)$ mice were separated by SDS-PAGE, transferred to nitrocellulose, and were probed with rabbit polyclonal anti-ACE antibody. Normal soluble somatic ACE was detected in wild-type and heterozygous mice at $160 \mathrm{kD}$, but not in homozygous mutant mice. A modified 85-kD form of ACE was found in heterozygous and homozygous mutant mice, but not in wild type. $(B)$ Proteins from the lungs, kidneys, and testes of the three genotypes were analyzed by Western blot as previously described (17). Wild-type and heterozygous mice contain the normal $160-\mathrm{kD}$ somatic ACE in lung and kidney and the 105-kD testis ACE isoform in the testis. Homozygous mutant mice do not contain any detectable ACE in any tissue. The modified $85 \mathrm{kD}$ ACE protein was not detected in any genotype, even upon overexposure of the blot (data not shown).

chloride towards certain substrates, whereas the amino-terminal domain is relatively chloride insensitive. This chloride activation is present in wild type plasma but abolished in homozygous mutant plasma using either HHL (Fig. 4 D) or HGG (data not shown) as a substrate. These results confirm that the modified ACE allele produces a somatic ACE isoform that contains only the amino-terminal domain.

ACE activity assays with various substrates yield widely disparate measures of the enzyme activity in the blood of homozygous mutant mice. Although these values provide useful in vitro information, they are not directly relevant to the physiologic role of ACE in vivo. The most physiologically appropriate measure of ACE activity is its ability to cleave angiotensin I to angiotensin II. With angiotensin I as a substrate, pooled plasma from homozygous mutant mice have $\sim 34 \%$ the activity of wild-type plasma in initial velocity experiments (Fig. $4 \mathrm{C}$ ). Individual plasma samples were also analyzed for their ability to cleave angiotensin I under non-zero (substrate limiting) reaction conditions. The relative activity of homozygous mutant mouse plasma was $\sim 75 \%$ of the activity of wildtype plasma $(1.81 \pm 0.7 \mathrm{nmol}$ angiotensin $\mathrm{I} / \mathrm{min}$ per $\mathrm{ml}[-/-]$ vs. $2.34+1.71 \mathrm{nmol}$ angiotensin $\mathrm{I} / \mathrm{min}$ per $\mathrm{ml}[+/+])$. In these experiments, $\sim 20 \%$ of the available angiotensin I was cleaved. These results demonstrate that the modified ACE protein in homozygous mutant mice can generate a significant amount of angiotensin II. 

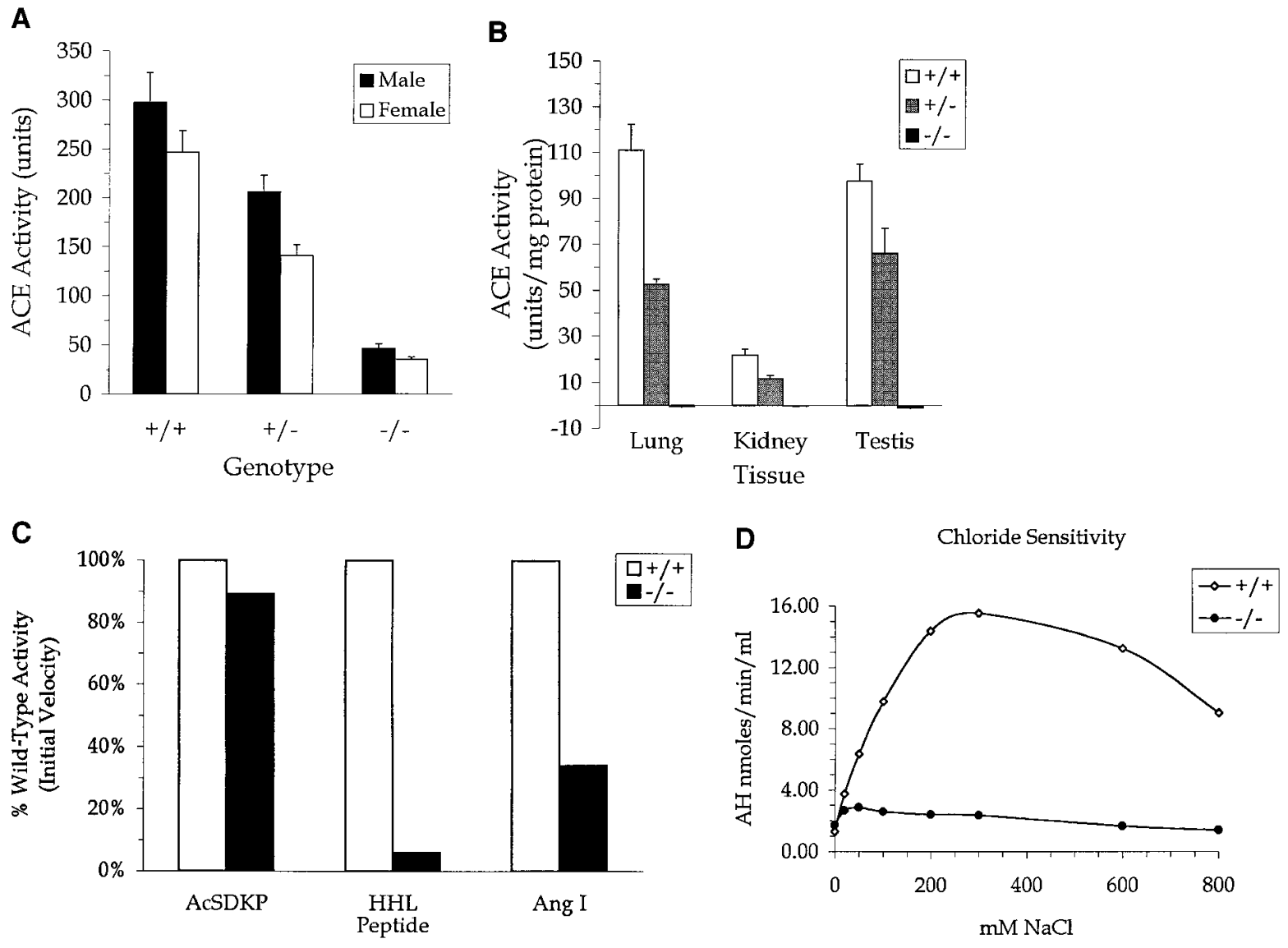

Figure 4. ACE activity assays. (A) Plasma was isolated from male and female wild-type $(+/+, n=7$ males and 6 females $)$, heterozygous $(+/-$, $n=7$ males and 6 females) and homozygous mutant $(-1-, n=7$ males and 5 females) mice, diluted 1:4 and analyzed for ACE activity using the artificial substrate HGG. This assay reveals both gender and genotype differences, with homozygous mutant mice having $\sim 15 \%$ of the activity of wild-type mice. $(B)$ Tissue from lung, kidney, and testis of male animals of the three genotypes $(n=4+/+, 3+/-$ and $3-/-)$ were homogenized in a detergent containing buffer and analyzed for ACE activity with HGG. ACE activity was reduced in heterozygotes and undetectable in all tissues from homozygous mutant mice. $(C)$ The plasma ACE activity of wild-type and homozygous mutant mice was tested against three substrates known to have different affinities for the $\mathrm{NH}_{2}$-terminal and $\mathrm{COOH}$-terminal domains of normal somatic ACE. Plasma from four mice of each genotype ( 2 male and 2 female) was pooled, and the initial velocity determined using either the $\mathrm{NH}_{2}$-terminal specific substrate AcSDKP, the relatively $\mathrm{COOH}$-terminal specific substrate $\mathrm{HHL}$ or angiotensin I. Results are expressed as percentage of the wild-type value. As expected, plasma from homozygous mutant mice contains much more activity against AcSDKP ( $89 \%$ of wild type) than HHL (6\%). (D) The influence of chloride on the hydrolysis of HHL was determined. Plasma from wild-type or homozygous mutant mice was dialyzed against distilled water and various chloride concentrations were added to aliquots of the dialyzed plasma. ACE activity toward HHL was determined as before. Plasma from wild-type $(+/+)$ mice shows a much greater activation by chloride than that from homozygous mutant $(-/-)$ animals, consistent with the known chloride insensitivity of the amino terminus of ACE (5).

Blood pressure. To test whether restoration of circulating ACE would affect blood pressure, the systolic blood pressure of wild-type, heterozygous, and homozygous mutant ACE. 2 mice were measured using a computerized tail-cuff system (Fig. 5). Homozygous mutant mice had significantly lower systolic pressures $(75 \mathrm{mmHg})$ than normal mice $(110 \mathrm{mmHg})$. Heterozygous mice were no different from wild type (110 $\mathrm{mmHg}$ ). However, heterozygous females did have somewhat lower average blood pressures $(95 \mathrm{mmHg})$. The reason for this is unknown. The low systolic blood pressures of the ACE. 2 homozygous mutant mice are not significantly different to those seen in completely ACE-deficient mice, suggesting that circulating ACE plays a relatively minor role in the control of blood pressure.

Renal structure and function. The kidneys from wild-type, heterozygous, and homozygous mutant mice were examined histologically. The kidneys from homozygous mutant mice exhibited vascular hypertrophy of small arteries and arterioles. The severity of this phenotype varied between normal vascular structure to marked vascular thickening. There was no apparent correlation between the degree of vascular thickening and the blood pressure or the age at death of the individual animals (data not shown). No vascular thickening was present in heterozygous animals.

It has previously been reported that animals lacking all ACE have a marked medullary and papillary atrophy (17). This was carefully examined in the ACE. 2 homozygous mutant mice. Both by visual inspection of the kidney and by microscopic examination, 14 of 18 animals presented with renal medullary and papillary development similar to wild-type mice (Fig. 6 A). One animal presented with unilateral calyceal dilatation, and three animals had calyceal dilatation similar to that 


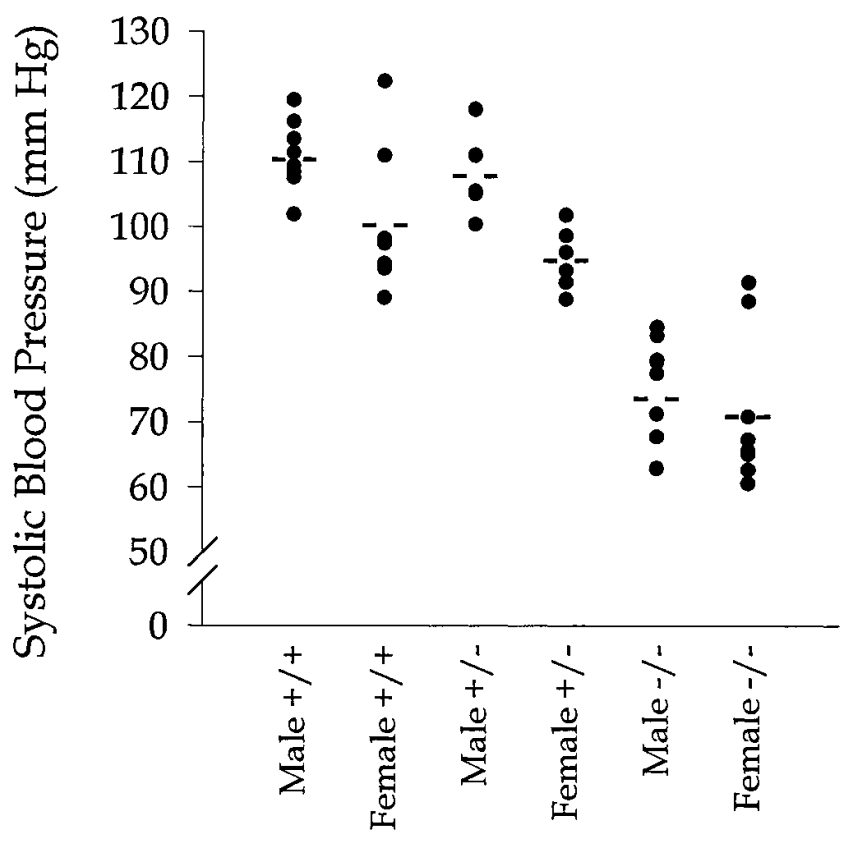

Gender and Genotype

Figure 5. Blood pressure. The systolic blood pressures of male and female wild-type $(+/+)$, heterozygous $(+/-)$, and homozygous mutant $(-/-)$ mice ( $n=7-9$ per group) were determined by a computerized tail-cuff system. Each data point represents the mean of $4 \mathrm{~d}$ of measurements taken after a 7-d training period. The average for each group is represented by a broken bar. Homozygous mutant mice had blood pressures $\sim 40 \mathrm{mmHg}$ lower than wild type $(P<0.01)$. No differences were seen between genders in any genotype except heterozygotes, in which the females had a $15-\mathrm{mmHg}$ lower blood pressure than the males $(P<0.05)$.

seen in completely ACE-deficient mice. Present in approximately half the ACE. 2 homozygous mutant mice is the focal expansion of Bowman's space (Fig. $6 B$ ). This pathology was seen in a small minority of glomeruli and was not associated with obvious distal nephron obstruction. Thus, whereas renal lesions were present in some of the ACE.2 homozygous mutant mice, many lack the severe renal pathology observed in mice lacking production of all ACE.

Mice totally deficient in ACE cannot effectively concentrate urine, consistent with the medullary hypoplasia present in the kidneys of these animals (17). Urinary excretion was examined in the ACE.2 strain by 24-h urine collections accompanied by water deprivation. Surprisingly, the homozygous mutant mice of the ACE. 2 strain had a urinary concentrating defect similar to that of mice totally deficient in the production of ACE. The ACE.2 homozygous mutants produced approximately twice the volume of urine as wild-type mice $(1,473 \pm 133$ $[-/-] \mu l$ vs. $691 \pm 75 \mu \mathrm{l}[+/+])$, Fig. $7 A)$. This urine was about half as concentrated as that of wild-type controls $(1,166 \pm 87$ $\mathrm{mosMol} / \mathrm{kg}[-/-]$ vs. $1,963 \pm 178 \mathrm{mosMol} / \mathrm{kg}[+/+]$, Fig. 7 B). Heterozygous mice were not different from normal in this respect. Significantly, the concentrating defect was present in homozygous mutant mice with normal renal medullary and papillary structure (average volume $1,143 \pm 141 \mu$, average os- molality $1,140 \pm 112 \mathrm{mosMol} / \mathrm{kg}, n=7$ animals). Unlike mice that lack ACE, the urinary concentrating defect in the ACE.2 homozygous mutant mice is not secondary to lack of a renal papilla. This finding provides evidence that the renin-angiotensin system plays a major physiologic role in the urinary concentrating mechanism of mice.

Fertility. The ACE.2 homozygous males do not express the testis isoform of ACE. We therefore predicted that they would exhibit the same male fertility defect found in male mice that are totally deficient in ACE. These completely ACE deficient animals sire litters that are significantly smaller than wild-type controls despite producing mature sperm that are normal in number, morphology, and motility (17).

To test male fertility in the ACE.2 strain, male wild-type, heterozygous or homozygous mutant mice were mated to superovulated wild-type C57BL/6 females. Homozygous mutant males sired significantly fewer litters ( 3 litters from 14 plugged females) than wild-type males (10 litters from 14 plugged females, $\left.\chi^{2}<0.001\right)$. Furthermore, the litters from the homozygous mutant males were significantly smaller than those from wild-type males $(P<0.001$, Fig. 8$)$. Thus, the ACE. 2 strain homozygous mutant mice exhibit reduced male fertility similar to that seen in mice totally deficient in ACE. Histologic examination of the testis from ACE. 2 homozygous mutant mice revealed no morphologic abnormalities (data not shown). This is similar to the observation of a normal histology in the testis of males totally deficient in the production of ACE. Female fertility appeared normal since both wild type and homozygous mutant females produced similar sized litters (average 4.3 pups/litter for both genotypes) when mated to C57BL/6J males.

\section{Discussion}

Previously, our group characterized the ACE.1 strain of mice, which have a complete absence of both circulating and tissue ACE (17). These animals have low systolic blood pressures, abnormalities in renal structure, the inability to concentrate urine, and reduced male fertility. Heterozygotes of the ACE.1 strain bear one unaltered ACE gene and contain 60\% the wild-type levels of plasma and tissue ACE protein as determined by ACE activity measurement. Interestingly, these animals are phenotypically similar to wild-type mice demonstrating that the effects of ACE deletion are not dominant.

The ACE. 2 strain of mice described in this paper bear an unusual genetic lesion that results in the formation of a modified somatic ACE protein containing only the amino-terminal catalytic domain. This domain has been shown to be catalytically active against the known physiological ACE substrates (5). Indeed, plasma from the ACE.2 homozygous mutant animals generates angiotensin II from angiotensin I at a rate that is $34 \%$ normal under zero order reaction conditions. The levels are even higher (75\%) under non-zero reaction conditions when substrate is limiting. However, because the modified ACE lacks the carboxy-terminal membrane anchor, this protein is entirely secreted from cells. As a result, tissue ACE activity is absent. The ACE. 2 homozygous mutant mice are in many ways physiologically similar to the ACE.1 homozygous mutant mice completely lacking both circulating and tissue ACE (17). Both strains of mice have very low systolic blood pressures that are not statistically different from each other. Both are also unable to concentrate urine effectively. The 

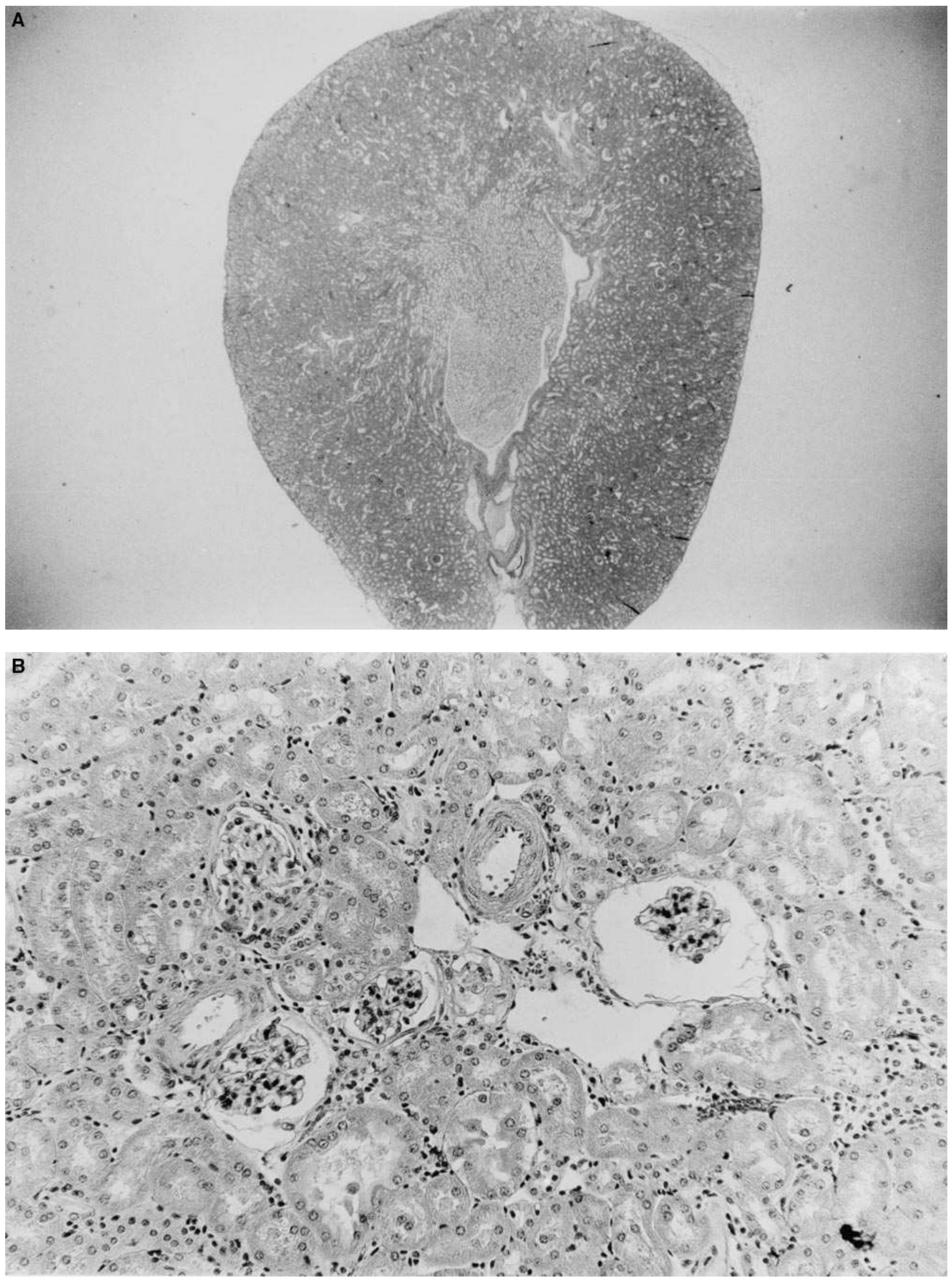

Figure 6. Renal histology. $(A)$ A low power view $(50 \times)$ of a kidney from a homozygous mutant mouse reveals that the renal medulla and papilla are intact. $(B)$ Higher power view $(250 \times)$ shows enlargement of Bowman's spaces and vascular thickening of an arteriole. 
A

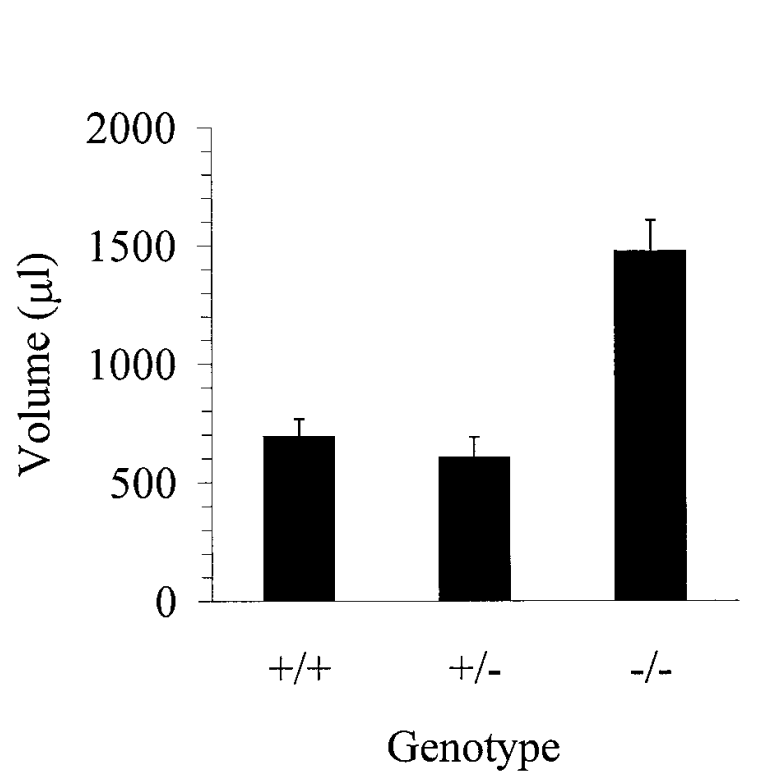

Genotype

Urine Volume
B

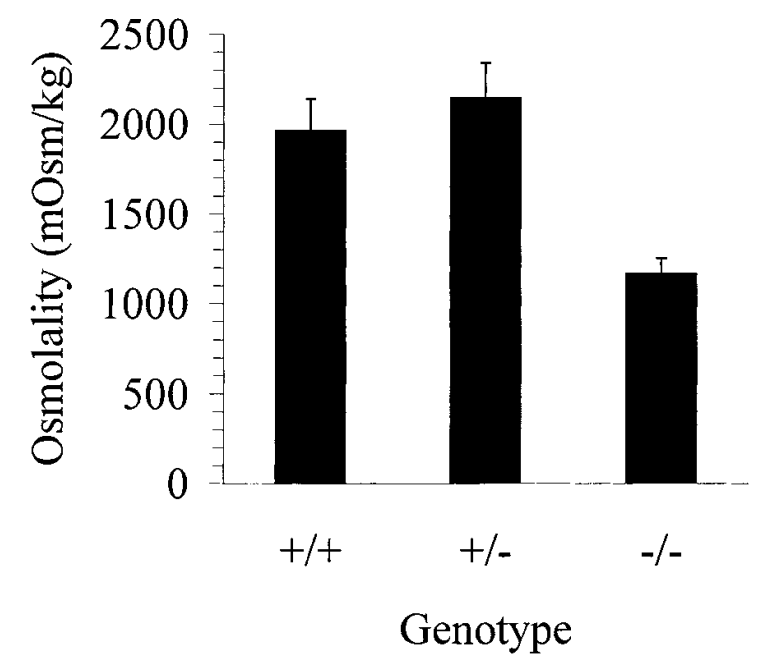

Figure 7. Urinalysis. Urine was collected from wild-type $(+/+)$, heterozygous $(+/-)$, and homozygous mutant $(-/-)$ mice $(n=11-13$ per group) for $24 \mathrm{~h}$ with water removed $6 \mathrm{~h}$ before the collection. $(A)$ Total urine volume was approximately twice as large in homozygous mutant mice as in either wild type or heterozygotes $(P<0.001)$. (B) The homozygous mutant mice produce urine that is half as concentrated as wild-type or heterozygous mice $(P<0.002)$.

ACE.1 and the ACE.2 strains of mice lack testis ACE, and therefore it is not surprising that both strains show an equivalent defect in male fertility. A significant difference between the two strains is the degree of renal pathology. ACE.1 animals present with medullary and papillary atrophy that is often apparent by 1 mo of age (unpublished observations). In contrast, many of the ACE. 2 homozygous mutant animals contain a preserved renal medulla and papilla even at 9 mo of age. This

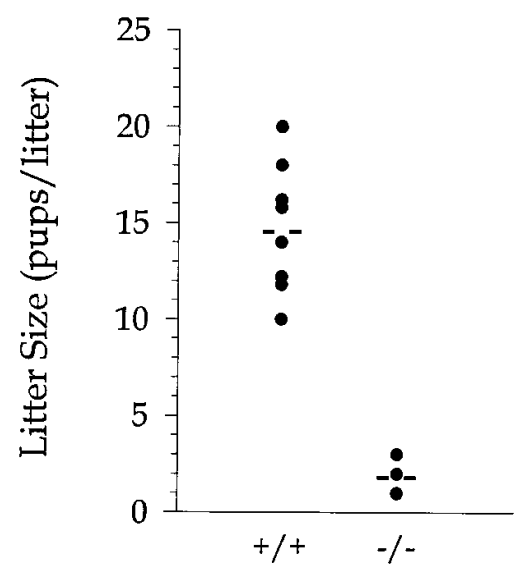

Paternal Genotype
Figure 8. Male fertility. The fertility of male wild-type $(+/+)$ and homozygous mutant $(-1-)$ mice was determined. Males were individually housed and bred overnight to single superovulated females. The females were checked for copulatory plugs in the morning. The female mice were killed 2 wk after copulation, and the number of pups were counted. Homozygous mutant males sire significantly fewer litters than wild type $\left(\chi^{2}<0.001\right)$, each of which contains significantly fewer pups per litter $(P<0.001)$.

difference is unlikely to be due to strain variation, since both lines of mice were generated in the same lab using the same ES cells and breeder strains. Thus, although the homozygous mutant ACE.2 mice contain plasma ACE activity approaching that of ACE. 1 heterozygotes, the ACE. 2 mice exhibit much of the phenotype observed in the ACE.1 homozygous mutant animals lacking all ACE. This strongly suggests that circulating ACE is not sufficient for a functional renin-angiotensin system.

The abnormalities present in the homozygous mutant ACE. 2 mice are similar to those described for mice completely lacking $\mathrm{ACE}$ and for mice that lack angiotensinogen, the precursor to angiotensin II $(16,17,23,24)$. This suggests that the ACE.2 homozygous mutant mice cannot generate sufficient angiotensin II despite significant plasma ACE activity; a finding consistent with research indicating that the bulk of angiotensin II formation occurs in the tissues such as the lung (11). This study is also consistent with the concept of a local reninangiotensin system. The components of the renin-angiotensin system exist in many organs and are postulated to regulate tissue specific angiotensin II production (25). These local systems depend critically on the presence of tissue-bound ACE and are therefore nonfunctional in the ACE. 2 homozygous mutants. ACE also plays a major role in the metabolism of bradykinin, and some of the phenotype may be due to increased levels of this vasodilator.

The difference in renal histology between the ACE. 2 and ACE.1 underscores the role of ACE and the renin-angiotensin system in kidney development. It has previously been reported that mice completely lacking ACE develop renal lesions that involve blunting of the renal papilla and associated dilatation of the renal calyces (17). These lesions are almost certainly due to lack of angiotensin II production since they also occur in mice that lack angiotensinogen and in rats treated neonatally with $\mathrm{AT}_{1}$ receptor antagonists $(23,24,26)$. It is not clear whether the renal abnormalities are due directly to a requirement for angiotensin II in normal renal development, or if they are secondary to the low blood pressure or increased urine flow present in these animals. Indeed, Brattleboro rats 
are unable to concentrate urine due to antidiuretic hormone deficiency and have an increased incidence of hydronephrotic lesions similar to those described for ACE-deficient mice (27). In contrast, many ACE. 2 homozygous mutant mice have low blood pressure and defects in urinary concentration without marked medullary atrophy. This finding suggests that secondary effects are not solely responsible for this renal lesion. The ACE.2 homozygous mutant mice have a significant amount of circulating ACE activity, and this enzyme level may be sufficient to rescue the medullary and papillary malformations but not the other physiological phenotypes in some instances.

The findings from the ACE. 2 strain provide insight into the role of the renin-angiotensin system in urinary concentration mechanisms. Previous studies of urinary concentrating ability in ACE or angiotensinogen deficient mice were complicated by the renal abnormalities present in these animals. The present work with the ACE.2 strain mice suggests that the reninangiotensin system plays a direct role in urinary concentration. The homozygous mutants produce an inappropriately dilute urine despite preservation of the renal papilla. These results are consistent with several studies that support a physiological role for the RAS in urine concentration. Direct injections of angiotensin II into the renal artery induce increases in urine osmolality (28), and mice that lack the $\mathrm{AT}_{1 \mathrm{~A}}$ receptor produce dilute urine despite a normal renal architecture (29). Several authors have suggested that the renin-angiotensin system is required to produce the low papillary blood flow necessary for maintenance of the normal renal concentration gradient (28, 30). However, we cannot yet distinguish between this mechanism and other possibilities, such as hemodynamically mediated reductions in the delivery of solute to the loop of Henle. It is interesting to note that treatment of adult humans or animals with ACE inhibitors does not cause changes in urine osmolality. This discrepancy is likely due to differences between the transient and incomplete blockade of tissue ACE achieved with an inhibitor and the permanent and complete loss of tissue ACE produced in our homozygous mutant mice.

The findings presented here further support a role for ACE in male fertility. Unlike the other physiologic abnormalities, this defect is not secondary to a lack of angiotensin II since angiotensinogen deficient male mice have been reported as having normal fertility (23). Although we strongly suspect that the testis isoform of ACE is important in male fertility, we cannot exclude the possibility that changes in blood pressure or renal function are responsible for the reduced fertility observed in the ACE. 2 homozygous mutant males.

In summary, we report the construction and characterization of mice containing a truncated form of somatic ACE. These animals have $\sim 34 \%$ the ACE activity in plasma but completely lack tissue ACE. The physiology of these animals is very similar to that of mice completely deficient in ACE and stands in sharp contrast to the normal physiology of heterozygous ACE.1 animals containing reduced plasma ACE activity. These observations strongly support the concept that tissuebound ACE activity is critical in the control of normal blood pressure and the ability of mice to concentrate urine effectively.

\section{Acknowledgments}

The authors would like to thank Kristen Thomas for her technical assistance and Nancy Bradley for her histological preparations. We would also like to thank Sunamita Tuple and Hsinchen Jean Lin for technical assistance. Charles Esther is a Medical Woodruff Fellow. Tom Howard is an Emory University Pathology resident and the 1994 ASIP Pathologist-In-Training Award recipient.

This research was supported by National Institutes of Health grants DK-39777, DK-44280, DK-45215, and DK-51445.

\section{References}

1. Corvol, P., T.A. Willaims, and F. Soubrier. 1995. Peptidyl dipeptidase A: angiotensin I-converting enzyme. Methods Enzymol. 248:283-305.

2. Bernstein, K.E., and B.C. Berk. 1993. The biology of angiotensin II receptors. Am. J. Kidney Dis. 2:745-754.

3. Soubrier, F., F. Alhenc-Gelas, C. Hubert, J. Allegrini, M. John, G. Tregear, and P. Corvol. 1988. Two putative active centers in human angiotensin I-converting enzyme revealed by molecular cloning. Proc. Natl. Acad. Sci. USA. 85:9386-9390.

4. Bernstein, K.E., B.M. Martin, A.S. Edwards, and E.A. Bernstein. 1989. Mouse angiotensin I-converting enzyme is a protein composed of two homologous domains. J. Biol. Chem. 264:11945-11951.

5. Wei, L., F. Alhenc-Gelas, P. Corvol, and E. Clauser. 1991. The two homologous domains of the human angiotensin I-converting enzyme are both catalytically active. J. Biol. Chem. 266:9002-9008.

6. Jaspard, E., L. Wei, and F. Alhenc-Gelas. 1993. Differences in the properties and enzymatic specificities of the two active sites of angiotensin I-converting enzyme (kininase II). Studies with bradykinin and other natural peptides. J. Biol. Chem. 268:9496-9503.

7. Ehlers, M.R.W., and J.F. Riordan. 1991. Angiotensin converting enzyme: zinc- and inhibitor-binding stiochiometries of the somatic and testis isozymes. Biochemistry. 30:7118-7126.

8. Rieger, K.J., N. Saez-Servent, M.P. Papet, J. Wdzieczak-Bakala, J.L. Morgat, J. Thierry, W. Voelter, and M. Lenfant. 1993. Involvement of human plasma angiotensin I-converting enzyme in the degradation of the haemoregulatory peptide N-acetyl-seryl-aspartyl-lysyl-proline. Biochem. J. 296:373-378.

9. Azizi, M., A. Rousseau, E. Ezan, T.T. Guyene, S. Michelet, J.M. Grognet, M. Lenfant, P. Corvol, and J. Menard. 1996. Acute angiotensin-converting enzyme inhibition increases the plasma level of the natural stem cell regulator N-acetyl-seryl-aspartyl-lysyl-proline. J. Clin. Invest. 97:839-844.

10. Beldent, V., A. Michaud, L. Wei, M.T. Chauvet, and P. Corvol. 1993. Proteolytic release of human angiotensin-converting enzyme. Localization of the cleavage site. J. Biol. Chem. 268:26428-26434.

11. Ng, K.K., and J.R. Vane. 1967. Conversion of angiotensin I to angiotensin II. Nature (Lond.). 216:762-766.

12. Waeber, B., J. Nussberger, L. Juillerat, and H.R. Brunner. 1989. Angiotensin converting enzyme inhibition: discrepancy between antihypertensive effect and suppression of enzyme activity. J. Cardiovasc. Pharmacol. 14(Suppl. 4):S53-S59.

13. Berecek, K.H., S.J. King, and J.N. Wu. 1993. Angiotensin-converting enzyme and converting enzyme inhibitors. In Cellular and Molecular Biology of the Renin-Angiotensin System. M.K. Raizada, M.I. Phillips, and C. Sumners, editors. CRC Press, Boca Raton. 183-220.

14. Howard, T.E., S.-Y. Shai, K.G. Langford, B.M. Martin, and K.E. Bernstein. 1990. Transcription of testicular angiotensin-converting enzyme (ACE) is initiated within the 12th intron of the somatic ACE gene. Mol. Cell. Biol. 10: 4294-4302.

15. Langford, K.G., S.-Y. Shai, T.E. Howard, M.J. Kovac, P.A. Overbeek, and K.E. Bernstein. 1991. Transgenic mice demonstrate a testis specific promoter for angiotensin converting enzyme (ACE). J. Biol. Chem. 266:1555915562.

16. Krege, J.H., S.W.M. John, L.L. Langenbach, J.B. Hodgin, J.R. Hagaman, E.S. Bachman, J.C. Jennette, D.A. O'Brien, and O. Smithies. 1995. Malefemale differences in fertility and blood pressure in ACE-deficient mice. Nature (Lond.). 375:146-148.

17. Esther, C.R., Jr., T.E. Howard, E.M. Marino, J.M. Goddard, M.R. Capecchi, and K.E. Bernstein. 1996. Mice lacking angiotensin-converting enzyme have low blood pressure, renal pathology and reduced male fertility. Lab. Invest. 74:953-965.

18. Mansour, S.L., K.R. Thomas, and M.R. Capecchi. 1988. Disruption of the proto-oncogene int-2 in mouse embryo-derived stem cells: a general strategy for targeting mutations to non-selected genes. Nature (Lond.). 336:348-352.

19. Cushman, D., and H. Cheung. 1971. Spectrophotometric assay and properties of the angiotensin-converting enzyme of rabbit lung. Biochem. Pharmacol. 20:1637-1648.

20. Chirgwin, J.M., A.E. Przybyla, R.J. MacDonald, and W.J. Rutter. 1979. Isolation of biologically active ribonucleic acid from sources enriched in ribonuclease. Biochemistry. 18:5294-5299.

21. Langford, K.G., Y. Zhou, L.D. Russell, J.N. Wilcox, and K.E. Bernstein. 1993. Regulated expression of testis angiotensin-converting enzyme during spermatogenesis in mice. Biol. Reprod. 48:1210-1218.

22. Krege, J.H., J.B. Hodgin, J.R. Hagaman, and O. Smithies. 1995. A non- 
invasive computerized tail-cuff system for measuring blood pressure in mice. Hypertension (Dallas). 25:1111-1115.

23. Kim, H.-S., J.H. Krege, K.D. Kluckman, J.R. Hagaman, J.B. Hodgin, C.F. Best, J.C. Jennette, T.M. Coffman, N. Maeda, and O. Smithies. 1995. Genetic control of blood pressure and the angiotensinogen locus. Proc. Natl. Acad. Sci. USA. 92:2735-2739.

24. Nimura, F., P.A. Labosky, J. Kakuchi, S. Okubo, H. Yoshida, T. Oikawa, T. Ichiki, A.J. Naftilan, A. Fogo, T. Inagami, et al. 1995. Gene targeting in mice reveals a requirement for angiotensin in the development and maintenance of kidney morphology and growth factor regulation. J. Clin. Invest. 96 : 2947-2954.

25. Swales, J.D. 1993 A perspective on extrarenal renin. In The ReninAngiotensin System. J. Ian, S. Robertson, and M.G. Nicholls, editors. Gower Medical Publishing, London. 48.1-48.14.

26. Friberg, P., B. Sundelin, S.-O. Bohman, A. Bobik, H. Nilsson, A. Wich- man, H. Gustafsson, J. Petersen, and M.A. Adams. 1994. Renin-angiotensin system in neonatal rats: induction of a renal abnormality in response to ACE inhibition or angiotensin II antagonism. Kidney Int. 45:485-492.

27. Henderson, I.W., J.A. Oliver, C.M. Milne, and R.J. Balment. 1982. Incidence and characteristics of hydronephrosis in Brattleboro rats. Ann. NY Acad. Sci. 394:21-29.

28. Faubert, P.H., S.-Y. Chou, and J.G. Porush. 1987. Regulation of papillary plasma flow by angiotensin II. Kidney Int. 32:472-478.

29. Ito, M., M.I. Oliverio, P.J. Mannon, C.F. Best, N. Maeda, O. Smithies, and T.M. Coffman. Regulation of blood pressure by the type $1 \mathrm{~A}$ angiotensin II receptor gene. 1995. Proc. Natl. Acad. Sci. USA. 92:3521-3525.

30. Hansell, P., M. Sjoquitst, and H.R. Ulfendahl. 1988. Effect of a converting enzyme inhibitor on vasa recta blood flow in rat kidney. Am. J. Physiol. 254: F492-F499. 